Research Article

\title{
Upper Semicontinuous Property of Uniform Attractors for the 2D Nonautonomous Navier-Stokes Equations with Damping
}

\author{
Xin-Guang Yang and Jun-Tao Li \\ College of Mathematics and Information Science, Henan Normal University, Xinxiang, \\ Henan 453007, China
}

Correspondence should be addressed to Xin-Guang Yang; yangxinguang@hotmail.com

Received 25 May 2013; Revised 20 August 2013; Accepted 21 August 2013

Academic Editor: Ahmed El-Sayed

Copyright (C) 2013 X.-G. Yang and J.-T. Li. This is an open access article distributed under the Creative Commons Attribution License, which permits unrestricted use, distribution, and reproduction in any medium, provided the original work is properly cited.

Our aim is to investigate the long-time behavior in terms of upper semicontinuous property of uniform attractors for the 2D nonautonomous Navier-Stokes equations with linear damping and nonautonomous perturbation external force, that is, the convergence of corresponding attractors when the perturbation tends to zero.

\section{Introduction}

In the present paper, we investigate the long-time behavior of uniform attractors for the nonautonomous 2D NavierStokes equations with damping and singular external force that governs the motion of incompressible fluid

$$
u_{t}-v \Delta u+(u \cdot \nabla) u+\alpha u+\nabla p=f_{0}(t, x)+\varepsilon^{-\rho} f_{1}\left(\frac{t}{\varepsilon}, x\right)
$$

$$
\begin{gathered}
\operatorname{div} u=0, \\
\left.u(t, x)\right|_{\partial \Omega}=0, \\
u(\tau, x)=u_{\tau}(x),
\end{gathered}
$$

where $x \in \Omega \subset \mathbb{R}^{2}$ is a bounded domain with smooth boundary $\partial \Omega, v$ is the kinematic viscosity of the fluid, $u=$ $u(t, x)=\left(u_{1}(t, x), u_{2}(t, x)\right)$ is the velocity vector field which is unknown, $p$ is the pressure, $\alpha>0$ is positive constant, $t \in \mathbb{R}_{\tau}=[\tau,+\infty)$, and $\varepsilon$ is a small positive parameter.
Along with (1)-(4), we consider the averaged NavierStokes equation with damping

$$
\begin{gathered}
u_{t}-v \Delta u+(u \cdot \nabla) u+\alpha u+\nabla p=f_{0}(t, x), \\
\nabla \cdot u=0, \\
\left.u(t, x)\right|_{\partial \Omega}=0, \\
u(\tau, x)=u_{\tau}(x),
\end{gathered}
$$

formally corresponding to the case $\varepsilon=0$.

The function

$$
f^{\varepsilon}(x, t)= \begin{cases}f_{0}(x, t)+\varepsilon^{-\rho} f_{1}\left(x, \frac{t}{\varepsilon}\right), & 0<\varepsilon<1, \\ f_{0}(x, t), & \varepsilon=0\end{cases}
$$

represents the external forces of problem (1)-(4) for $\varepsilon>0$ and problem (5)-(8) for $\varepsilon=0$, respectively. 
The functions $f_{0}(x, s)$ and $f_{1}(x, s)$ are taken from the space $L_{b}^{2}(\mathbb{R} ; H)$ of translational bounded functions in $L_{\text {loc }}^{2}(\mathbb{R} ;$ $H)$, namely,

$$
\begin{aligned}
& \left\|f_{0}\right\|_{L_{b}^{2}}^{2}:=\sup _{t \in \mathbb{R}} \int_{t}^{t+1}\left\|f_{0}(s)\right\|^{2} d s=M_{0}^{2}, \\
& \left\|f_{1}\right\|_{L_{b}^{2}}^{2}:=\sup _{t \in \mathbb{R}} \int_{t}^{t+1}\left\|f_{1}(s)\right\|^{2} d s=M_{1}^{2},
\end{aligned}
$$

for some constants $M_{0}, M_{1} \geq 0$.

We denote

$$
Q^{\varepsilon}= \begin{cases}M_{0}+2 M_{1} \varepsilon^{-\rho}, & 0<\varepsilon<1, \\ M_{0}, & \varepsilon=0,\end{cases}
$$

and note that $Q^{\varepsilon}$ is of the order $\varepsilon^{-\rho}$ as $\varepsilon \rightarrow 0^{+}$.

As a straightforward consequence of (9), we have

$$
\left\|f^{\varepsilon}\right\|_{L_{b}^{2}} \leq Q^{\varepsilon}
$$

When $\alpha=0$ in (5)-(8), the system reduces to the wellknown 2D incompressible Navier-Stokes equation:

$$
\begin{gathered}
u_{t}-v \Delta u+(u \cdot \nabla) u+\nabla p=f, \\
\nabla \cdot u=0 .
\end{gathered}
$$

Since the last century, the global well-posedness and large-time behavior of solutions to the Navier-Stokes equations have attracted many mathematicians to study. For the well posedness of 3D incompressible Navier-Stokes equations, in 1934, Leray $[1,2]$ derived the existence of weak solution by weak convergence method; Hopf [3] improved Leray's result and obtained the familiar Leray-Hopf weak solution in 1951. Since the Navier-Stokes equations lack appropriate priori estimate and the strong nonlinear property, the existence of strong solution remains open. For the infinite-dimensional dynamical systems, Sell [4] constructed the semiflow generated by the weak solution which lacks the global regularity and obtained the existence of global attractor of the incompressible Navier-Stokes equations on any bounded smooth domain; Cheskidov and Foias [5] introduced a weak global attractor with respect to the weak topology of the natural phase space for 3D Navier-Stokes equation with periodic boundary; Flandoli and Schmalfuß [6] deduced the existence of weak solutions and attractors for 3D NavierStokes equations with nonregular force; Kloeden and Valero [7] investigated the weak connection of the attainability set of weak solutions of 3D Navier-Stokes equations; Cutland [8] obtained the existence of global solutions for the 3D NavierStokes equations with small samples and germs; Chepyzhov and Vishik [9-11] investigated the trajectory attractors for 3D nonautonomous incompressible Navier-Stokes system which is based on the works of Leray and Hopf. Using the weak convergence topology of the space $H$ (see below for the definition), Kapustyan and Valero [12] proved the existence of a weak attractor in both autonomous and nonautonomous cases and gave an existence result of strong attractors. Kapustyan et al. [13] considered a revised 3D incompressible
Navier-Stokes equations generated by an optimal control problem and proved the existence of pullback attractors by constructing a dynamical multivalued process. For more results of the well-posedness and long-time behavior of the $2 \mathrm{D}$ autonomous incompressible Navier-Stokes equations, such as the existence of global solutions, the existence of global attractors, Hausdorff dimension, and inertial manifold approximation, we can refer to Ladyzhenskaya [14], Robinson [15], Sell and You [16], and Temam [17, 18]. Moreover, Caraballo and Real [19] derived the existence of global attractor for 2D autonomous incompressible Navier-Stokes equation with delays; Chepyzhov and Vishik [20, 21] investigated the longtime behavior and convergence of corresponding uniform (global) attractors for the 2D Navier-Stokes equation with singularly oscillating forces as the external force tend to be steady state by virtue of linearization method and estimate the corresponding difference equations; Foias and Temam $[22,23]$ gave a survey about the geometric properties of solutions and the connection between solutions, dynamical systems, and turbulence for Navier-Stokes equations, such as the existence of $\omega$-limit sets; Rosa [24] and Hou and Li [25] obtained the existence of global (uniform) attractors for the 2D autonomous (nonautonomous) incompressible NavierStokes equations in some unbounded domain, respectively; $\mathrm{Lu}$ et al. [26] and $\mathrm{Lu}$ [27] proved the existence of uniform attractors for 2D nonautonomous incompressible NavierStokes equations with normal or less regular normal external force by establishing a new dynamical systems framework; Miranville and Wang [28] derived the attractors for nonautonomous nonhomogeneous Navier-Stokes equations.

However, the infinite-dimensional systems for 3D incompressible Navier-Stokes equations have not been yet completely resolved, so many mathematicians pay attention to this challenging problem. In this regard, some mathematicians pay their attentions to the Navier-Stokes equation with damping. Let us recall some known results for the $3 \mathrm{D}$ incompressible Naver-Stokes equations with damping. For the 3D autonomous Navier-Stokes equation with damping, the authors of [29] showed that the initial boundary value problem of a 3D Navier-Stokes equation with damping has a unique weak solution and Song and Hou [30] derived the global attractors for the same autonomous system. Kalantarov and Titi [31] investigated the Navier-Stokes-Voight equations as an inviscid regularization of the 3D incompressible NavierStokes equations, and further obtained the existence of global attractors for Navier-Stokes-Voight equations. Recently, Qin et al. [32] showed the existence of uniform attractors by uniform condition-(C) and weak continuous method to obtain uniformly asymptotical compactness in $H^{1}$ and $H^{2}$. However, there are fewer results for the upper semicontinuous and lower semicontinuous for the nonautonomous system with perturbation case. In this paper, we will show the long-time behavior in terms of upper semicontinuous property of uniform attractors for the problem (1)-(4), that is, the convergence of corresponding attractors when the perturbation tends to zero.

This paper is organized as follows: in Section 2, we will give some preliminaries of uniform attractors; in Section 3, the uniform boundedness of uniform attractors of $2 \mathrm{D}$ 
Navier-Stokes equation with damping for $\varepsilon \geq 0$ will be obtained; the main result will be stated in the last section.

\section{Some Preliminaries of Uniform Attractors}

The Hausdorff semidistance in $X$ from one set $B_{1}$ to another set $B_{2}$ is defined as

$$
\operatorname{dist}_{X}\left(B_{1}, B_{2}\right)=\sup _{b_{1} \in B_{1}} \inf _{b_{2} \in B_{2}}\left\|b_{1}-b_{2}\right\|_{X}
$$

$L^{p}(\Omega)(1 \leq p \leq+\infty)$ is the generic Lebesgue space and $H^{s}(\Omega)$ is the usual Sobolev space. We set $E:=\{u \mid u \in$ $\left.\left(C_{0}^{\infty}(\Omega)\right)^{2}, \operatorname{div} u=0\right\}, H$ is the closure of the set $E$ in $\left(L^{2}(\Omega)\right)^{2}$ topology with norm $\|\cdot\|$ or $\|\cdot\|_{H}, V$ is the closure of the set $E$ in $\left(H_{0}^{1}(\Omega)\right)^{2}$ topology, and $W$ is the closure of the set $E$ in $\left(H_{0}^{2}(\Omega)\right)^{2}$ topology.

The family of functions $L_{\text {loc }}^{2}(\mathbb{R} ; H)$ denote a local Bochner integration function class, and $L_{b}^{2}(\mathbb{R} ; H)$ denotes all translation bounded functions which satisfies

$$
\sup _{t \in \mathbb{R}} \int_{t}^{t+1}\|\sigma(s, x)\|_{H}^{2} d s<+\infty
$$

for all $\sigma \in L_{\text {loc }}^{2}(\mathbb{R} ; H)$; that is, $\sigma$ is translation bounded in $L_{\text {loc }}^{2}(\mathbb{R} ; H) . L_{\text {tc }}^{2}(\mathbb{R} ; H)$ is translation compact function in $L^{2}(\mathbb{R} ; H)$. Obviously, $L_{b}^{2}(\mathbb{R} ; H) \subset L_{\text {loc }}^{2}(\mathbb{R} ; H)$.

Operator $P$ is the Helmholtz-Leray orthogonal projection in $\left(L^{2}(\Omega)\right)^{2}$ onto the space $H, A:=-P \Delta$ is the Stokes operator subject to the nonslip homogeneous Dirichlet boundary condition with the domain $\left(H^{2}(\Omega)\right)^{2} \cap V, A$ is a self-adjoint positively defined operator on $H$ with domain $D(A)=$ $\left(H^{2}(\Omega)\right)^{2} \cap V$, and $\lambda>0$ is the first eigenvalue for the Stokes operator $A$; we define the Hilbert space $H^{\sigma}$ as $H^{\sigma}=D\left(A^{\sigma / 2}\right)$ with its inner product $(u, v)_{H^{\sigma}}=\left(A^{\sigma / 2} u, A^{\sigma / 2} v\right)_{\left(L^{2}(\Omega)\right)^{2}}$ and norm topology as $\|u\|_{H^{\sigma}}=\left\|A^{\sigma / 2} u\right\|_{\left(L^{2}(\Omega)\right)^{2}}$.

The problems (1)-(4) and (5)-(8) can be written as a generalized abstract form

$$
\begin{gathered}
u_{t}+v A u+\alpha u+B(u, u)=\sigma(t, x) \\
\operatorname{div} u=0 \\
\left.u\right|_{\partial \Omega}=0 \\
u(\tau, x)=u_{\tau}
\end{gathered}
$$

where the pressure $p$ has disappeared by force of the application of the Leray-Helmholtz projection $P$, and $B(u, v)=$ $(u \cdot \nabla) v$ is the bilinear operator. The bilinear form $B(\cdot, \cdot)$ can be extended as a continuous trilinear operator $b(u, v, w)=$ $(B(u, v), w)$ and satisfies

$$
\begin{gathered}
b(u, v, v)=0, \quad \forall u, v, w \in V, \\
b(u, v, w)=-b(u, w, v), \quad \forall u, v, w \in V, \\
\|b(u, v, w)\| \leq C\|u\|^{1 / 2}\|u\|_{1}^{1 / 2}\|v\|_{1}\|w\|_{1}, \quad \forall u, v, w \in V,
\end{gathered}
$$

$$
\begin{gathered}
\|b(u, v, u)\| \leq C\|u\|^{1 / 2}\|u\|_{1}^{3 / 2}\|v\|_{1}, \quad \forall u, v \in V \\
\|b(u, v, w)\| \leq C\|u\|_{1}\|v\|_{1}\|w\|^{1 / 2}\|w\|_{1}^{1 / 2}, \quad \forall u, v, w \in V \\
\|b(u, v, w)\| \leq C \lambda_{1}^{1 / 4}\|u\|_{1}\|v\|_{1}\|w\|_{1}, \quad \forall u, v, w \in V, \\
\|b(u, v, w)\| \leq C\|u\|^{1 / 2}\|A u\|^{1 / 2}\|v\|_{V}\|w\|, \\
\forall(u, v, w) \in D(A) \times V \times H .
\end{gathered}
$$

Firstly, we will give some Lemmas which can be found in [20], then derive some new results to prove the uniform boundedness of corresponding attractors in Section 3.

Lemma 1. For each $\tau \in \mathbb{R}$, every nonnegative locally summable function $\phi$ on $\mathbb{R}_{\tau}$ and every $\beta>0$, one has

$$
\int_{\tau}^{t} \phi(s) e^{-\beta(t-s)} d s \leq \frac{1}{1-e^{-\beta}} \sup _{\theta \geq \tau} \int_{\theta}^{\theta+1} \phi(s) d s,
$$

for all $t \geq \tau$.

Proof. See, for example, Chepyzhov et al. [20].

Lemma 2. Let $\zeta: \mathbb{R}_{\tau} \rightarrow \mathbb{R}^{+}$fulfill the fact that for almost every $t \geq \tau$, the differential inequality

$$
\frac{d}{d t} \zeta(t)+\phi_{1}(t) \zeta(t) \leq \phi_{2}(t)
$$

where, for every $t \geq \tau$, the scalar functions $\phi_{1}$ and $\phi_{2}$ satisfy

$$
\int_{\tau}^{t} \phi_{1}(s) d s \geq \beta(t-\tau)-\gamma, \quad \int_{t}^{t+1} \phi_{2}(s) d s \leq M,
$$

for some $\beta>0, \gamma \geq 0$, and $M \geq 0$. Then

$$
\zeta(t) \leq e^{\gamma} \zeta(\tau) e^{-\beta(t-\tau)}+\frac{M e^{\gamma}}{1-e^{-\beta}}, \quad \forall t \geq \tau .
$$

Proof. See, for example, Chepyzhov et al. [20].

The existence of global solution and uniform attractor for (17)-(20) can be derived by similar methods as [33].

Theorem 3. (1) Assume $\sigma \in L_{\text {loc }}^{2}(\mathbb{R} ; H), u_{\tau} \in H$; then problem (17)-(20) possesses a unique global weak solution $u(t, x)$ which satisfies

$$
u \in C([\tau,+\infty) ; H) \cap L^{2}(\tau, T ; V) \cap L^{4}\left(\tau, T ;\left(L^{4}(\Omega)\right)^{2}\right) .
$$

Moreover, one chooses an arbitrary nonautonomous external force $\sigma_{0}(t, x) \in L_{b}^{2}(\mathbb{R} ; H)$ and fixed, the global solution $u(t, x)$ generates a process $\left\{U_{\sigma}(\tau, t)\right\}(\tau \in \mathbb{R}, t>\tau, \sigma \in \Sigma)$ which is continuous with respect to $u_{\tau}$, where $\sigma$ is a symbol which belongs to the symbol space $\Sigma=\mathscr{H}\left(\sigma_{0}\right)=\left[\left\{\sigma_{0}(s+h) \mid h \epsilon\right.\right.$ $\mathbb{R}\}]_{L_{\mathrm{loc}}^{2}(\mathbb{R}, H)}$, and $[\cdot]_{E}$ means the closure in the topology $E$.

(2) Assume that $u_{\tau} \in H, \sigma \in \Sigma \subset L_{\text {loc }}^{2}([\tau,+\infty] ; H)$; then the family of processes $\left\{U_{\sigma}(t, \tau), t \geq \tau \in \mathbb{R}\right\},\left(\sigma \in \mathscr{H}\left(\sigma_{0}\right)\right)$ generated by the global weak solution of problem (17)-(20) possesses a uniform (with respect to $\sigma \in \Sigma=\mathscr{H}\left(\sigma_{0}\right)$ ) attractor $\mathscr{A}_{\mathscr{H}\left(\sigma_{0}\right)}=\mathscr{A}_{\Sigma}$ in $H$. 
Theorem 4. Assume that $u_{\tau} \in H$; the functions $f_{0}(x, s)$ and $f_{1}(x, s)$ are taken from the space $L_{b}^{2}(\mathbb{R}, H)$ of translational bounded functions in $L_{\mathrm{loc}}^{2}(\mathbb{R} ; H)$ and (10)-(13) hold, and then the family of processes $\left\{U_{f^{\varepsilon}}(t, \tau), t \geq \tau, t, \tau \in \mathbb{R}\right\}$ generated by the global solution of problem (1)-(4) possesses uniform (with respect to $\left.\sigma=f^{\varepsilon} \in \Sigma\right)$ attractors $\mathscr{A}^{\varepsilon}$ for any fixed $\varepsilon \in(0,1)$ in $H$.

Proof. As the similar argument in [33], we choose $\sigma(t, x)=$ $f^{\varepsilon}(t, x)$ in [33], since $f_{0}$ and $f_{1}$ are translational bounded in $L_{\text {loc }}^{2}(\mathbb{R} ; H)$, and then for any fixed $\varepsilon \in(0,1]$, we can deduce that $f^{\varepsilon}(t, x)$ is translational bounded in $L_{\text {loc }}^{2}(\mathbb{R} ; H)$ and the existence of uniformly compact attractors $\mathscr{A}^{\varepsilon}$ for any fixed $\varepsilon \in(0,1)$.

Theorem 5. If the function $f_{0}(t, x)$ is taken from the space $L_{b}^{2}(\mathbb{R} ; H)$ of translational bounded functions in $L_{\mathrm{loc}}^{2}(\mathbb{R} ; H)$, then the processes $\left\{U_{f_{0}}(t, \tau), t \geq \tau, t, \tau \in \mathbb{R}\right\}$ generated by system (5)-(8) have a uniformly (with respect to $\sigma=f_{0} \in \Sigma$ ) compact attractor $\mathscr{A}^{0}$ in $\mathrm{H}$.

Proof. As the similar technique in [33], we can easily deduce the existence of a uniformly compact attractor $\mathscr{A}^{0}$ if we choose $\sigma(t, x)=f_{0}(t, x)$ since $f^{0}$ is translation bounded in $L_{\text {loc }}^{2}(\mathbb{R} ; H)$.

The structure of the uniform attractor will be discussed as follows: since the functions $f_{0}(t)$ and $f_{1}(t)$ are translation bounded and satisfy (10)-(13), the global solution of problem (1)-(4) generates the family of processes $\left\{U^{\varepsilon}(t, \tau), t \geq \tau, \tau \in\right.$ $\mathbb{R}\}$ acting on $H$ by the formula $U^{\varepsilon}(t, \tau) u_{\tau}^{\varepsilon}=u^{\varepsilon}(t), t \geq \tau$, where $u^{\varepsilon}(t)$ is a solution to (1)-(4).

Similar to the procedure in [33] and by Theorem 4, the processes class $\left\{U^{\varepsilon}(t, \tau)\right\}$ has a uniformly (with respect to $t \epsilon$ $\mathbb{R})$ absorbing set

$$
B^{\varepsilon}:=\left\{u^{\varepsilon} \in H \mid\left\|u^{\varepsilon}\right\|_{H} \leq C Q^{\varepsilon}\right\}
$$

which is bounded in $H$ for any fixed $\varepsilon \in(0,1)$, which means that for any bounded set $B \subset H$, there exists a time $T=$ $T\left(\varepsilon, B^{\varepsilon}\right)$ such that

$$
U^{\varepsilon}(t, \tau) B \subseteq B^{\varepsilon}, \quad \forall \tau \in \mathbb{R}, \forall t \geq \tau+T .
$$

Hence,

$$
\begin{aligned}
B_{1}^{\varepsilon} & :=\bigcup_{\tau \in R} U^{\varepsilon}(\tau+1, \tau) B^{\varepsilon}, \\
B_{2}^{\varepsilon} & :=\bigcup_{\tau \in R} U^{\varepsilon}(\tau+2, \tau) B^{\varepsilon}, \\
& \vdots \\
B_{[T]}^{\varepsilon} & :=\bigcup_{\tau \in R} U^{\varepsilon}(\tau+[T], \tau) B^{\varepsilon}
\end{aligned}
$$

are also uniformly absorbing with respect to $\sigma(x, t)$ as $f^{\varepsilon}$ or $f^{0}$ which belongs to $\Sigma$, $[T]$ is the integer part of $T$.
The processes $\left\{U^{\varepsilon}(t, \tau)\right\}$ have a uniform global attractor as uniform $\omega$-set

$$
\mathscr{A}^{\varepsilon}=\omega(\widetilde{B}):=\bigcap_{h>0} \overline{\left[\bigcup_{t-\tau \geq h} U^{\varepsilon}(t, \tau) \widetilde{B}\right]_{H}},
$$

where $\overline{[\cdot]}_{H}$ denotes the closure in $H$ and $\widetilde{B}$ is an arbitrarily uniformly bounded absorbing set of the processes $\left\{U^{\varepsilon}(t, \tau)\right\}$; here, we can set $\widetilde{B}=B^{\varepsilon}$.

On the other hand, for each fixed $\varepsilon, \mathscr{A}^{\varepsilon}$ is also bounded in $H$, since $\mathscr{A}^{\varepsilon} \subseteq B_{i}^{\varepsilon}(i=1,2, \ldots,[T])$. Assuming $f_{0}, f_{1} \in$ $L_{\mathrm{tc}}^{2}(\mathbb{R} ; H)$, then $f^{\varepsilon}(t) \in L_{\mathrm{tc}}^{2}(\mathbb{R} ; H)$. Besides, if $\varepsilon>0$ and $\hat{f}^{\varepsilon} \in$ $\mathscr{H}\left(f^{\varepsilon}\right)$, then

$$
\widehat{f}^{\varepsilon}(t)=\widehat{f}_{0}(t)+\varepsilon^{-\rho} \widehat{f}_{1}\left(\frac{t}{\varepsilon}\right),
$$

for some $\widehat{f}_{0} \in \mathscr{H}\left(f_{0}\right)$ and $\widehat{f}_{1} \in \mathscr{H}\left(f_{1}\right)$.

Next, we consider the equation class as follows to describe the structure of the uniform attractor $\mathscr{A}^{\varepsilon}$

$$
\widehat{u}_{t}+\nu A \widehat{u}+\alpha \widehat{u}+B(\widehat{u})=\widehat{f}^{\varepsilon}(t), \quad \widehat{f}^{\varepsilon} \in \mathscr{H}\left(f^{\varepsilon}\right) .
$$

For every external force $\widehat{f}^{\varepsilon} \in \mathscr{H}\left(f^{\varepsilon}\right)$, by the wellposeness of the abstract equation (17), we can derive that (38) generates a family of processes $\left\{U_{\bar{f}^{\varepsilon}}(t, \tau)\right\}$ on $H$, which shares similar properties to $\left\{U^{\varepsilon}(t, \tau)\right\}$, corresponding to the original equation (1) with external force $f^{\varepsilon}(x, t)$. Moreover, from Theorem 3 we know the map

$$
\left(u_{\tau}, \hat{f}^{\varepsilon}\right) \longmapsto U_{\hat{f}^{\varepsilon}}(t, \tau) u_{\tau}
$$

is $\left(H \times \mathscr{H}\left(f^{\varepsilon}\right), H\right)$-continuous.

Definition 6. The kernel $\mathscr{K}_{\hat{f}^{\varepsilon}}$ of (17) is the family of all complete orbits $\{\widehat{u}(t), t \in R\}$ which are uniformly bounded in $H$. The set

$$
\mathscr{K}_{\hat{f}^{\varepsilon}}(\tau)=\left\{\widehat{u}(\tau) \mid \widehat{u} \in \mathscr{K}_{\hat{f}^{\varepsilon}}\right\} \subset H
$$

is called the kernel section of $\mathscr{K}_{\bar{f}^{\varepsilon}}$ at time $t=\tau$. For every $\varepsilon \in(0,1)$, the following representation (complete orbit) of uniform attractors $\mathscr{A}^{\varepsilon}$ of (1) holds:

$$
\mathscr{A}^{\varepsilon}=\bigcup_{\hat{f}^{\varepsilon} \in \mathscr{H}\left(f^{\varepsilon}\right)} \mathscr{K}_{\hat{f}^{\varepsilon}}(\tau) .
$$

Definition 7. The structure of uniform attractors for problem (5)-(8) can be described as the uniform $\omega$-set or kernel section:

$$
\begin{aligned}
& \mathscr{A}^{0}=\omega\left(\widetilde{B}_{0}\right):=\bigcap_{h>0} \overline{\left[\bigcup_{t-\tau \geq h} U^{0}(t, \tau) \widetilde{B}_{0}\right]}, \\
& \mathscr{A}^{0}=\bigcup_{\hat{f}_{0} \in \mathscr{H}\left(f_{0}\right)} \mathscr{K}_{\widehat{f}_{0}}(\tau) .
\end{aligned}
$$




\section{Uniform Boundedness of $\mathscr{A}^{\varepsilon}$ in $H$}

Firstly, we consider the auxiliary linear equation with nonautonomous external force $K(t)$ and give some useful estimates and then prove the uniform boundedness of $\mathscr{A}^{\varepsilon}$ in $H$.

Considering the linear equation

$$
Y_{t}+\nu A Y+\alpha Y=K(t),\left.\quad Y\right|_{t=\tau}=0
$$

we obtain the following lemmas.

Lemma 8. Assume $K \in L_{b}^{2}(\mathbb{R} ; V) \subset L_{\text {loc }}^{2}(\mathbb{R} ; V) \subset L_{\text {loc }}^{2}(\mathbb{R} ; H)$; then problem (43) has a unique solution

$$
\begin{gathered}
Y \in L^{2}\left((\tau, T) ;\left(H^{3}(\Omega)\right)^{2}\right) \cap C((\tau, T) ; W), \\
\partial_{t} Y \in L^{2}\left((\tau, T) ; W^{\prime}\right) .
\end{gathered}
$$

Moreover, the following inequalities

$$
\begin{gathered}
\|Y(t)\|_{V}^{2} \leq C \int_{\tau}^{t} e^{-(C / v)(t-s)}\|K(s)\|_{H}^{2} d s, \\
\|Y(t)\|_{W}^{2} \leq C \int_{\tau}^{t} e^{-(C / v)(t-s)}\|K(s)\|_{V}^{2} d s, \\
\int_{t}^{t+1}\|Y(t)\|_{H}^{2} d s \leq C\left(\|Y(t)\|_{H}^{2}+\int_{t}^{t+1}\|K(s)\|_{H}^{2} d s\right), \\
\int_{t}^{t+1}\|Y(s)\|_{W}^{2} d s \leq C\left(\|Y(t)\|_{V}^{2}+\int_{t}^{t+1}\|K(s)\|_{H}^{2} d s\right), \\
\int_{t}^{t+1}\|Y(s)\|_{H^{3}}^{2} d s \leq C\left(\|Y(t)\|_{W}^{2}+\int_{t}^{t+1}\|K(s)\|_{V}^{2} d s\right)
\end{gathered}
$$

hold for every $t \geq \tau$ and some constant $C=C(\lambda)>0$, independent of the initial time $\tau \in R$.

Proof. Firstly, similar to the discussion in [32] or [34], by the Galerkin approximation method, we can obtain the existence of global solution; here we omit the details.

Then, multiplying (43) by $Y, A Y$, and $A^{2} Y$, respectively, using the Poincaré inequality, we get

$$
\begin{aligned}
\frac{1}{2} \frac{d}{d t}\|Y\|^{2}+\nu\|\nabla Y\|^{2}+\alpha\|Y\|^{2} & =(K(t), Y) \\
& \leq \frac{2}{\alpha}\|K(t)\|^{2}+\frac{\alpha}{2}\|Y\|^{2}, \\
\frac{1}{2} \frac{d}{d t}\|\nabla Y\|^{2}+\nu\|A Y\|^{2}+\alpha\|\nabla Y\|^{2} & =(K(t), A Y) \\
& \leq \frac{1}{v}\|K(t)\|^{2}+\nu\|A Y\|^{2},
\end{aligned}
$$

$$
\begin{aligned}
\frac{1}{2} \frac{d}{d t}\|\nabla Y\|^{2}+v\|A Y\|^{2}+\alpha\|\nabla Y\|^{2} & =(K(t), A Y) \\
& \leq \frac{2}{v}\|K(t)\|^{2}+\frac{v}{2}\|A Y\|^{2},
\end{aligned}
$$

$$
\begin{aligned}
\frac{1}{2} \frac{d}{d t}\|A Y\|^{2}+v\left\|A^{3} Y\right\|^{2}+\alpha\|A Y\|^{2} & =\left(K(t), A^{2} Y\right) \\
& \leq \frac{C}{v}\|K(t)\|_{V}^{2}+\frac{v}{2}\left\|A^{3} Y\right\|^{2} .
\end{aligned}
$$

By the Gronwall inequality to (51), (53), integrating over $(t, t+1)$ for (50), (52), and (53), we can easily complete the proof.

Setting $K(t, \tau)=\int_{\tau}^{t} k(s) d s, t \geq \tau, \tau \in \mathbb{R}$, we have the following lemma.

Lemma 9. Let $k \in L_{\text {loc }}^{2}(\mathbb{R}, H)$. Assume that

$$
\sup _{t \geq \tau, \tau \in \mathbb{R}}\left\{\|K(t, \tau)\|_{H}^{2}+\int_{t}^{t+1}\|K(s, \tau)\|_{V}^{2} d s\right\} \leq l^{2}
$$

holds for some constant $l \geq 0$. Then the solution $y(t)$ to the following Cauchy problem

$$
y_{t}+v A y+\alpha y=k\left(\frac{t}{\varepsilon}\right),\left.\quad y\right|_{t=\tau}=0
$$

with $\varepsilon \in(0,1)$ satisfies the inequality

$$
\|y(t)\|_{H}^{2}+\int_{t}^{t+1}\|y(s)\|_{V}^{2} d s \leq C l^{2} \varepsilon^{2}, \quad \forall t \geq \tau,
$$

where constant $C>0$ is independent of $K$.

Proof. Noting that

$$
K_{\varepsilon}(t)=\int_{\tau}^{t} k\left(\frac{s}{\varepsilon}\right) d s=\varepsilon \int_{\tau / \varepsilon}^{t / \varepsilon} k(s) d s=\varepsilon K\left(\frac{t}{\varepsilon}, \frac{\tau}{\varepsilon}\right)
$$

and then using (54) and (57), we can deduce the following estimates of $K_{\varepsilon}(t)$ as

$$
\begin{aligned}
\sup _{t \geq \tau}\left\|K_{\varepsilon}(t)\right\|_{H} \leq C l \varepsilon \\
\int_{t}^{t+1}\left\|K_{\varepsilon}(s)\right\|_{H}^{2} d s \leq \int_{t}^{t+1}\left\|K_{\varepsilon}(s)\right\|_{V}^{2} d s \\
=\varepsilon^{2} \int_{t}^{t+1}\left\|K\left(\frac{s}{\varepsilon}, \frac{\tau}{\varepsilon}\right)\right\|_{V}^{2} d s \\
\leq C \varepsilon^{2} \sup _{t \geq \tau}\left\{\int_{t}^{t+1}\|K(s, \tau)\|_{V}^{2} d s\right\} \leq C l^{2} \varepsilon^{2}
\end{aligned}
$$


From Lemmas 2 and 8 , we have

$$
\begin{aligned}
& \int_{\tau}^{t} e^{-(C / v)(t-s)}\left\|K_{\varepsilon}(s)\right\|_{H}^{2} d s \\
& \leq \int_{t-1}^{t} e^{-(C / v)(s-t)}\left\|K_{\varepsilon}(s)\right\|_{H}^{2} d s \\
& \quad+\int_{t-2}^{t-1} e^{-(C / v)(s-t)}\left\|K_{\varepsilon}(s)\right\|_{H}^{2} d s+\cdots \\
& \leq \int_{t-1}^{t}\left\|K_{\varepsilon}(s)\right\|_{H}^{2} d s+e^{-(C / v)} \int_{t-2}^{t-1}\left\|K_{\varepsilon}(s)\right\|_{H}^{2} d s \\
& \quad+e^{-2(C / v)} \int_{t-3}^{t-2}\left\|K_{\varepsilon}(s)\right\|_{H}^{2} d s+\cdots \\
& \leq\left(1+e^{-(C / v)}+e^{-2(C / v)}+\cdots\right)\left\|K_{\varepsilon}(s)\right\|_{L_{b}^{2}(\mathbb{R} ; H)}^{2} \\
& \leq \frac{1}{\left(1-e^{-(C / v)}\right)}\left\|K_{\varepsilon}(s)\right\|_{L_{b}^{2}(\mathbb{R} ; H)}^{2} \\
& \leq \frac{1}{\left(1-e^{-(C / v)}\right)} \sup _{t \geq \tau} \int_{t}^{t+1}\left\|K_{\varepsilon}(s)\right\|_{H}^{2} d s \\
& \leq \frac{1}{\lambda\left(1-e^{-(C / v)}\right)} \sup _{t \geq \tau}^{2} \int_{t}^{t+1}\left\|K_{\varepsilon}(s)\right\|_{V}^{2} d s
\end{aligned}
$$

Similarly, we derive that

$$
\int_{\tau}^{t} e^{-(C / v)(t-s)}\left\|K_{\varepsilon}(s)\right\|_{V}^{2} d s \leq C l^{2} \varepsilon^{2} .
$$

Hence, using the Poincaré inequality, by (45)-(47) and (58)-(60), we derive

$$
\begin{gathered}
\|Y(t)\|_{V}^{2} \leq C l^{2} \varepsilon^{2} \\
\int_{t}^{t+1}\|Y(s)\|_{H}^{2} d s \leq C\left(\|Y(t)\|_{H}^{2}+\int_{t}^{t+1}\left\|K_{\varepsilon}(s)\right\|_{H}^{2} d s\right) \\
\leq C l^{2} \varepsilon^{2} \\
\int_{t}^{t+1}\|Y(s)\|_{W}^{2} d s \leq C\left(\|Y(t)\|_{V}^{2}+\int_{t}^{t+1}\left\|K_{\varepsilon}(s)\right\|_{H}^{2} d s\right) \\
\leq C l^{2} \varepsilon^{2}
\end{gathered}
$$

Next, we set

$$
Y(t)=\int_{\tau}^{t} y(s) d s
$$

which implies that for any $t \geq \tau$,

$$
\partial_{t} Y(t)=y(t)=\int_{\tau}^{t} \partial_{t} y(s) d s
$$

since $y(\tau)=0$ in $(55)$.
Integrating (55) with respect to time from $\tau$ to $t$, we see that $Y(t)$ is a solution to the problem

$$
\partial_{t} Y(t)+v A Y(t)+\alpha Y(t)=K_{\varepsilon}(t),\left.\quad Y(t)\right|_{t=\tau}=0,
$$

such that we can deduce that

$$
\begin{aligned}
& \|Y(t)\|_{H}^{2}+\|\nabla Y(t)\|_{H}^{2}+\int_{t}^{t+1}\|Y(s)\|_{H}^{2} d s \\
& =\|Y(t)\|_{V}^{2}+\int_{t}^{t+1}\|Y(s)\|_{H}^{2} d s \leq C l^{2} \varepsilon^{2}
\end{aligned}
$$

from (62) to (63).

Using (46) and (61), we conclude

$$
\|Y(s)\|_{W}^{2} \leq C l^{2} \varepsilon^{2} .
$$

Noting that $y(t)=\partial_{t} Y(t),(A Y(t), Y(t)) \sim\|Y(t)\|_{V}^{2}$, and $(A Y(t), A Y(t)) \sim\|Y(t)\|_{W}$, using (58), (68), and (69), we derive that

$$
\begin{aligned}
\left\|\partial_{t} Y(t)\right\|_{H}^{2} & =\|y(t)\|_{H}^{2} \\
& \leq C\left(\nu\|Y(t)\|_{W}^{2}+\alpha\|Y(t)\|_{H}^{2}+\left\|K_{\varepsilon}(t)\right\|_{H}^{2}\right) \leq C l^{2} \varepsilon^{2} .
\end{aligned}
$$

Hence, by (51), (58), and (62), we conclude

$$
\begin{aligned}
\int_{t}^{t+1}\|y(s)\|_{V}^{2} d s & =\int_{t}^{t+1}\left\|\frac{d}{d s} Y(s)\right\|_{V}^{2} \\
& \leq 2 \alpha\|Y\|_{V}^{2}+2 C\left\|K_{\varepsilon}(s)\right\|_{H}^{2} \leq C l^{2} \varepsilon^{2} .
\end{aligned}
$$

Combining (70) and (71), the proof for the lemma is finished.

Now, we will use the auxiliary linear equation and some estimates to prove the uniform boundedness of $\mathscr{A}^{\varepsilon}$ in $H$. For convenience, we set

$$
F_{1}(t, \tau)=\int_{\tau}^{t} f_{1}(s) d s, \quad t \geq \tau
$$

and assume

$$
\sup _{t \geq \tau, \tau \in \mathbb{R}}\left\{\left\|F_{1}(t, \tau)\right\|^{2}+\int_{t}^{t+1}\left\|F_{1}(s, \tau)\right\|_{V}^{2} d s\right\} \leq l^{2},
$$

for some constants $l \geq 0$ since $f_{0}(s)$ and $f_{1}(s)$ are translation bounded in $L_{\text {loc }}^{2}(\mathbb{R} ; V) \subset L_{\text {loc }}^{2}(\mathbb{R} ; H)$.

Theorem 10. The attractors $\mathscr{A}^{\varepsilon}$ of problem (1)-(4) with $\varepsilon \in$ $(0,1)$ (or $(5)-(8)$ with $\varepsilon=0)$ are uniformly (with respect to $\varepsilon$ ) bounded in $H$, namely,

$$
\sup _{\varepsilon \in[0,1)}\left\|\mathscr{A}^{\varepsilon}\right\|_{H}<+\infty
$$

Proof. Let $u^{\varepsilon}(t)=U^{\varepsilon}(t, \tau) u_{\tau}^{\varepsilon}$ be the solution to (1)-(4) with the initial data as $u_{\tau}^{\varepsilon} \in H$. For $\varepsilon>0$, we consider the auxiliary linear equation

$$
v_{t}+v A v+\alpha v=\varepsilon^{-\rho} f_{1}\left(\frac{t}{\varepsilon}\right),\left.\quad v\right|_{t=\tau}=0
$$


By Lemma 9, we have the estimate

$$
\|v(t)\|_{H}^{2}+\int_{t}^{t+1}\|v(s)\|_{V}^{2} d s \leq C l^{2} \varepsilon^{2(1-\rho)}, \quad \forall t \geq \tau .
$$

Multiplying (75) with $A v$ and integrating over $\Omega$, using the boundary value condition, we derive that

$$
\begin{aligned}
\frac{d}{d t} \| \nabla & v\left\|^{2}+2 \alpha\right\| \nabla v\left\|^{2}+2 v\right\| A v \|^{2} \\
& =\left(\varepsilon^{-\rho} f_{1}\left(\frac{t}{\varepsilon}\right), A v\right) \\
& \leq \frac{4}{v}\left\|\varepsilon^{-\rho} f_{1}\left(\frac{t}{\varepsilon}\right)\right\|_{H}^{2}+2 v\|A v\|^{2} .
\end{aligned}
$$

By the Gronwall inequality and similar to (60), noting that when $t$ tends to infinite, we can set $e^{-\alpha(t-\tau)}<\varepsilon^{2}$ such that

$$
\begin{aligned}
& \|v\|_{V}^{2} \\
& \leq C\|\nabla v\|_{H}^{2} \\
& \leq e^{-2 \alpha(t-\tau)}\|\nabla v(\tau)\|_{H}^{2}+\frac{4}{v} \int_{\tau}^{t} e^{-2 \alpha(t-s)}\left\|\varepsilon^{-\rho} f_{1}\left(\frac{s}{\varepsilon}\right)\right\|_{H}^{2} d s \\
& \leq e^{-2 \alpha(t-\tau)}\|\nabla v(\tau)\|_{H}^{2}+\frac{4}{v} \int_{\tau}^{t} e^{-2 \alpha(t-\tau)}\left\|\varepsilon^{-\rho} f_{1}\left(\frac{s}{\varepsilon}\right)\right\|_{H}^{2} d s \\
& \leq e^{-2 \alpha(t-\tau)}\|\nabla v(\tau)\|_{H}^{2}+\frac{4}{v} e^{-\alpha(t-\tau)} \int_{\tau}^{t} e^{-\alpha(t-\tau)}\left\|\varepsilon^{-\rho} f_{1}\left(\frac{s}{\varepsilon}\right)\right\|_{H}^{2} d s \\
& \leq C \varepsilon^{4}+C \varepsilon^{2(1-\rho)} \\
& \approx C \varepsilon^{2(1-\rho)},
\end{aligned}
$$

since $\varepsilon \in(0,1)$.

Setting the function $w(t)$ as

$$
w(t)=u(t)-v(t)
$$

which satisfies the problem

$$
w_{t}+v A w+\alpha w+B(w+v, w+v)=f_{0},\left.\quad w\right|_{t=\tau}=u_{\tau},
$$

where $u(t)$ is a solution for problem (1)-(4), and $v(t)$ is a solution to (75), $B(u, v)$ is the bilinear operator which is defined in Section 2.
Taking the scalar product of (80) with $w$ in $H$, we obtain

$$
\begin{aligned}
\frac{1}{2} \frac{d}{d t} & \|w\|^{2}+v\|w\|_{V}^{2}+\alpha\|w\|^{2}+(B(w+v, w+v), w) \\
= & \frac{1}{2} \frac{d}{d t}\|w\|^{2}+v\|w\|_{V}^{2}+\alpha\|w\|^{2}+b(w+v, w+v, w) \\
= & \frac{1}{2} \frac{d}{d t}\|w\|^{2}+v\|w\|_{V}^{2}+\alpha\|w\|^{2}+b(w+v, v, w) \\
& +b(w+v, w, w) \\
= & \frac{1}{2} \frac{d}{d t}\|w\|^{2}+v\|w\|_{V}^{2}+\alpha\|w\|^{2}+b(w, v, w)+b(v, v, w) \\
= & \left(f_{0}, w\right) .
\end{aligned}
$$

Here we use the property of trilinear operator (21)-(22); we observe that

$$
\begin{gathered}
|b(w, v, w)| \leq C\|w\|\|w\|_{V}\|v\|_{V} \leq \frac{v}{2}\|w\|_{V}^{2}+C\|w\|^{2}\|v\|_{V}^{2}, \\
|b(v, v, w)| \leq C\|v\|^{1 / 2}\|v\|_{W}^{1 / 2}\|v\|_{V}\|w\| \\
\leq\|v\|_{V}^{2}\|w\|^{2}+C\|v\|_{H}\|v\|_{W},
\end{gathered}
$$

so that

$$
\begin{aligned}
|b(w+v, v, w)| \leq & v\|w\|_{V}^{2}+C\|w\|^{2}\|v\|_{V}^{2} \\
& +\|v\|_{V}^{2}\|w\|^{2}+C\|v\|_{H}\|v\|_{W} .
\end{aligned}
$$

Moreover,

$$
\left(f_{0}, w\right) \leq \frac{\nu}{2}\|w\|_{V}^{2}+C\left\|f_{0}\right\|^{2} .
$$

Inserting (82)-(84) into (81) and then using the inequality

$$
\|v(t)\|^{2}=\|v(t)\|_{V}^{2} \leq C l^{2} \varepsilon^{2(1-\rho)}, \quad \forall t \geq \tau,
$$

and (78), we have

$$
\begin{aligned}
\frac{1}{2} \frac{d}{d t}\|w\|^{2}+v\|w\|_{V}+\alpha\|w\|^{2} \\
\leq v\|w\|_{V}^{2}+C\|w\|^{2}\|v\|_{V}^{2}+\|v\|_{V}^{2}\|w\|^{2} \\
+C\|v\|_{H}\|v\|_{W}+C\left\|f_{0}\right\|^{2} \\
\leq v\|w\|_{V}^{2}+2 C l^{2} \varepsilon^{2(1-\rho)}\|w\|^{2} \\
+C\|v\|^{2}\|v\|_{V}^{2}+C\left\|f_{0}\right\|^{2}
\end{aligned}
$$

which implies that

$$
\frac{d}{d t}\|w\|_{H}^{2}+\phi_{1}\|w\|_{H}^{2} \leq \phi_{2},
$$

where

$$
\begin{gathered}
\phi_{1}(t) \equiv \alpha-2 C l \varepsilon^{(1-\rho)} \geq \alpha, \\
\phi_{2}(t) \equiv C\|v\|^{2}\|v\|_{V}^{2}+C\left\|f_{0}\right\|^{2} .
\end{gathered}
$$


Therefore, from Theorem 3, we derive from (88) that for any $t \geq \tau$,

$$
\begin{gathered}
\int_{\tau}^{t} \phi_{1}(s) d s \geq \alpha(t-\tau), \\
\int_{t}^{t+1} \phi_{2}(s) d s \leq C\left(M_{0}^{2}+l^{4}\right) .
\end{gathered}
$$

Applying Lemma 2 with $\zeta(t)=\|w\|^{2}, \beta=\alpha, \gamma=0, M=$ $C\left(M_{0}^{2}+l^{4}\right)$, we get

$$
\|w\|_{H}^{2} \leq C e^{-\alpha(t-\tau)}\left\|u_{\tau}\right\|^{2}+C\left(M_{0}^{2}+l^{4}\right), \quad \forall t \geq \tau .
$$

Recalling that $u=w+v$ and using (85) and (90), we end up with

$$
\|u(t)\|_{H}^{2} \leq\|w\|_{H}^{2}+\|v\|_{H}^{2} \leq C e^{-\alpha(t-\tau)}\left\|u_{\tau}\right\|^{2}+C\left(l^{4}+M_{0}^{2}\right)
$$

for all $t \geq \tau$.

Thus, for every $0<\varepsilon \leq \varepsilon_{0}$, the processes $\left\{U_{\varepsilon}(t, \tau)\right\}$ have an absorbing set

$$
B_{0}:=\left\{u \in H \mid\|u\|_{H}^{2} \leq 2 C\left(l^{2}+M_{0}^{2}\right)\right\} .
$$

On the other hand, if $\varepsilon_{0}<\varepsilon<1$, the processes $\left\{U_{\varepsilon}(t, \tau)\right\}$ also possess an absorbing set

$$
B^{\varepsilon_{0}}=\left\{u \in H \mid\|u\|_{H} \leq C Q_{\varepsilon_{0}}\right\} .
$$

In conclusion, for every $\varepsilon_{0} \in[0,1)$, the set

$$
B_{*}:=B_{0} \bigcup B^{\varepsilon_{0}}
$$

is an absorbing set for the processes $\left\{U_{\varepsilon}(t, \tau)\right\}$ which is independent of $\varepsilon$. Since $\mathscr{A}^{\varepsilon} \subset B_{*}$, (74) follows and hence the proof is finished.

\section{Convergence of $\mathscr{A}^{\varepsilon}$ to $\mathscr{A}^{0}$}

Next, we will study the difference of two solutions for (1) with $\varepsilon>0$ and (4) with $\varepsilon=0$, which share the same initial data. Denote

$$
u^{\varepsilon}(t):=U^{\varepsilon}(t, \tau) u_{\tau},
$$

with $u_{\tau}$ belonging to the absorbing set $B_{*}$ which can be found in Section 3. In particular, for $\varepsilon=0$, since $u_{\tau} \in B_{*}$, we obtain

$$
\left\|u^{0}(t)\right\|_{H}^{2}+\int_{t}^{t+1}\left\|u^{0}(s)\right\|_{V}^{2} d s \leq R_{0}^{2}
$$

for some $R_{0}=R_{0}(\rho)$, as the size of $B^{*}$ depends on $\rho$.

Lemma 11. For every $\varepsilon \in(0,1), \tau \in \mathbb{R}$, and $u_{\tau} \in B_{*}$, the difference

$$
w(t)=u^{\varepsilon}(t)-u^{0}(t),
$$

where $u^{\varepsilon}(0)=u^{0}(0)=u_{\tau}$ satisfies the estimate

$$
\|w(t)\|_{H} \leq D \varepsilon^{1-\rho} e^{R(t-\tau)}, \quad \forall t \geq \tau,
$$

for some positive constants $D=D(\rho, l)$ and $R=R(\rho, l)$, both independent of $\varepsilon>0$.
Proof. Since the difference $w(t)$ solves

$$
\begin{array}{r}
w_{t}+\alpha w+v A w+B\left(u^{\varepsilon}, u^{\varepsilon}\right)-B\left(u^{0}, u^{0}\right)=\varepsilon^{-\rho} f_{1}\left(\frac{\varepsilon}{t}\right), \\
\left.w\right|_{t=\tau}=0,
\end{array}
$$

the difference

$$
q(t)=w(t)-v(t)
$$

fulfills the Cauchy problem

$$
q_{t}+\alpha q+v A q+B\left(u^{\varepsilon}, u^{\varepsilon}\right)-B\left(u^{0}, u^{0}\right)=0,\left.\quad q\right|_{t=\tau}=0,
$$

where $v(t)$ is the solution to (75).

Taking inner product in $H$ of (101) with $q$, we obtain

$$
\begin{aligned}
& \frac{1}{2} \frac{d}{d t}\|q\|^{2}+\alpha\|q\|^{2}+v\|\nabla q\|^{2} \\
& +\left(B\left(u^{\varepsilon}, u^{\varepsilon}\right)-B\left(u^{0}, u^{0}\right), q\right)=0 .
\end{aligned}
$$

Noting

$$
\begin{aligned}
& B\left(u^{\varepsilon}, u^{\varepsilon}\right)-B\left(u^{0}, u^{0}\right) \\
& \quad=B\left(u^{0}, q+v\right)+B\left(q+v, u^{0}\right)+B(q+v, q+v),
\end{aligned}
$$

we derive

$$
\begin{aligned}
\left(B\left(u^{\varepsilon}, u^{\varepsilon}\right)-B\left(u^{0}, u^{0}\right), q\right) \\
=b\left(u^{0}, v, q\right)+b\left(q, u^{0}, q\right)+b\left(v, u^{0}, q\right) \\
\quad+b(q, v, q)+b(v, v, q) .
\end{aligned}
$$
(104).

Next, we estimate each term on the right-hand side of Applying (22) to (27), we find

$$
\begin{aligned}
\left|b\left(q, u^{0}, q\right)\right| & \leq C\|q\|_{V}\|q\|\left\|u^{0}\right\|_{1} \\
& \leq \frac{v}{4}\|\nabla q\|^{2}+C\|q\|^{2}\left\|u^{0}\right\|_{V}^{2} \\
|b(q, v, q)| & \leq C\|q\|_{V}\|q\|\|v\|_{V} \\
& \leq \frac{v}{4}\|\nabla q\|^{2}+C\|q\|^{2}\|v\|_{V}^{2}, \\
|b(v, v, q)| & \leq C\|q\|_{V}\|v\|\|v\|_{V} \\
\leq & \frac{v}{4}\|\nabla q\|^{2}+C\|v\|^{2}\|v\|_{V}^{2}, \\
\left|b\left(u^{0}, v, q\right)\right| & +\left|b\left(v, u^{0}, q\right)\right| \\
\leq 2 C\left\|u^{0}\right\|^{1 / 2}\left\|u^{0}\right\|_{0}^{1 / 2}\|v\|^{1 / 2}\|v\|_{V}^{1 / 2}\|q\|_{V} & \frac{v}{4}\|\nabla q\|^{2}+C u^{0}\|\| u^{0}\left\|_{V}\right\| v\|\| v \|_{V} .
\end{aligned}
$$


Hence, from (105) to (107), we obtain

$$
\begin{aligned}
& \left|\left(B\left(u^{\varepsilon}, u^{\varepsilon}\right)-B\left(u^{0}, u^{0}\right), q\right)\right| \\
& \leq v\|\nabla q\|^{2}+C\|q\|^{2}\left(\left\|u^{0}\right\|_{0}^{2}+\|v\|_{V}^{2}\right) \\
& \quad+C\|v\|^{2}\|v\|_{V}^{2}+C\left\|u^{0}\right\|\left\|u^{0}\right\|_{V}\|v\|\|v\|_{V} \\
& \equiv v\|\nabla q\|^{2}+h(t)\|q\|^{2}+f(t),
\end{aligned}
$$

where $\|v\|_{V}$ and $\left\|u^{0}\right\|_{V}$ satisfy (76) and (96), respectively, and

$$
\begin{gathered}
h(t)=C\left(\left\|u^{0}\right\|_{V}^{2}+\|v(t)\|_{V}^{2}\right), \\
f(t)=C l^{2} \varepsilon^{2(1-\rho)}\|v\|_{V}^{2}+C R_{0} l \varepsilon^{1-\rho}\left\|u^{0}(t)\right\|_{V}\|v(t)\|_{V} .
\end{gathered}
$$

Thus, it follows from (102) and (104) that

$$
\frac{1}{2} \frac{d}{d t}\|q\|^{2}+\alpha\|q\|^{2} \leq h(t)\|q\|^{2}+f(t) .
$$
get

Noting that $\|q(\tau)\|_{H}=0$, by the Gronwall inequality, we

$$
\|q\|^{2} \leq 2 \exp \left\{2 C \int_{\tau}^{t} h(s) d s\right\} \int_{\tau}^{t} f(s) d s .
$$

Moreover,

$$
\begin{aligned}
\int_{\tau}^{t} h(s) d s \leq C\left(l^{4}+R_{0}^{2}\right)(t-\tau+1) \\
\int_{\tau}^{t} f(s) d s=\int_{\tau}^{t}\left[C l^{2} \varepsilon^{2(1-\rho)}\|v\|_{V}^{2}\right. \\
\left.\quad+C R_{0} l \varepsilon^{1-\rho}\left\|u^{0}(t)\right\|_{V}\|v(t)\|_{V}\right] d s \\
\leq C l^{4} \varepsilon^{4(1-\rho)}(t-\tau+1) \\
+C R_{0} l \varepsilon^{(1-\rho)} \int_{\tau}^{t}\left\|u^{0}(s)\right\|_{V}\|v(s)\|_{V} d s \\
\leq C l^{4} \varepsilon^{4(1-\rho)}(t-\tau+1) \\
+C R_{0} l \varepsilon^{(1-\rho)}\left(\int_{\tau}^{t}\left\|u^{0}(s)\right\|_{V}^{2} d s\right)^{1 / 2} \\
\times\left(\int_{\tau}^{t}\|v(s)\|_{V}^{2} d s\right)^{1 / 2} \\
\leq C l^{4} \varepsilon^{4(1-\rho)}(t-\tau+1) \\
+C R_{0}^{2} l^{2} \varepsilon^{2(1-\rho)}(t-\tau+1) \\
\leq C \varepsilon^{2(1-\rho)}\left(l^{4}+C R_{0}^{2} l^{2}\right)(t-\tau+1) .
\end{aligned}
$$

Consequently,

$$
\begin{aligned}
\|q(t)\|_{H}^{2} \leq & C \varepsilon^{2(1-\rho)}\left(l^{4}+C R_{0}^{2} l^{2}\right)(t-\tau+1) \\
& \times e^{C(t-\tau+1)\left(l^{4}+R_{0}^{2}\right)} \\
\leq & C^{\prime} D_{1}^{2} \varepsilon^{2(1-\rho)} e^{2 R_{1}(t-\tau)}
\end{aligned}
$$

holds for some positive constants $D_{1}=D_{1}(\rho, l)$ and $R_{1}=$ $R_{1}(\rho, 1)$.

Finally, since $w=q+v$, using (76) to control $\|v\|_{H}$, we may obtain

$$
\begin{aligned}
\|w(t)\|_{H}^{2} & \leq C\left(\|q\|_{H}^{2}+\|v\|_{H}^{2}\right) \\
& \leq C^{\prime} D_{1}^{2} \varepsilon^{2(1-\rho)} e^{2 R_{1}(t-\tau)}+C l^{2} \varepsilon^{2(1-\rho)} \\
& \leq D^{2} \varepsilon^{2(1-\rho)} e^{2 R(t-\tau)},
\end{aligned}
$$

where $R$ is a positive constant.

Next, we want to generalize Lemma 11 to derive the convergence of corresponding uniform attractors. Let the external force in (38) be $\widehat{f}=\widehat{f}^{\varepsilon} \in \mathscr{H}\left(f^{\varepsilon}\right)$, then $\widehat{f}_{1} \in \mathscr{H}\left(f_{1}\right)$ satisfies inequality (73).

Define

$$
\widehat{G}_{1}(t, \tau)=\int_{\tau}^{t} \widehat{f}_{1}(s) d s, \quad t \geq \tau,
$$

and we have

$$
\sup _{t \geq \tau, \tau \in \mathbb{R}}\left\{\left\|\widehat{G}_{1}(t, \tau)\right\|_{H}^{2}+\int_{t}^{t+1}\|\widehat{G}(s, \tau)\|_{H}^{2} d s\right\} \leq l^{2} .
$$

For any $\varepsilon \in[0,1]$, we observe that $\widehat{u}^{\varepsilon}(t)=U_{\widehat{f}^{\varepsilon}}(t, \tau) y_{\tau}$ is a solution to (38) with external force $\widehat{f}^{\varepsilon}=\widehat{f}_{0}+\varepsilon^{-\rho} \widehat{f}_{1}(\cdot / \varepsilon) \epsilon$ $\mathscr{H}\left(f^{\varepsilon}\right)$ and $y_{\tau}\left(f^{\varepsilon}\right) \in B_{*}$. For $\varepsilon>0$, we investigate the property of the difference

$$
\widehat{w}(t)=\widehat{u}^{\varepsilon}(t)-\widehat{u}^{0}(t) .
$$

Lemma 12. The inequality

$$
\|\widehat{w}(t)\| \leq D \varepsilon^{1-\rho} e^{R(t-\tau)}, \quad \forall t \geq \tau
$$

holds; here $D$ and $R$ are defined as in Lemma 11.

Proof. As the similar discussion to the proof of Lemma 11, replacing $\widehat{u}^{\varepsilon}, \widehat{f}_{0}$, and $\widehat{f}_{1}$ by $u^{\varepsilon}, f_{0}$, and $f_{1}$, respectively, noting that (96) still holds for $\widehat{u}^{0}$, and the family $\left\{U_{\widehat{f}^{\varepsilon}}(t, \tau)\right\},\left(\widehat{f}^{\varepsilon} \in\right.$ $\left.\mathscr{H}\left(f^{\varepsilon}\right)\right)$, is $\left(H \times \mathscr{H}^{\varepsilon}\left(f^{\varepsilon}\right), H\right)$-continuous, and using (116) in place of (73), we can finally complete the proof of the lemma.

The main result of this paper reads as follows.

Theorem 13. Let $f_{0}, f_{1} \in L_{t c}^{2}(\mathbb{R} ; H) \subset L_{b}^{2}(\mathbb{R} ; H)$, and let (73) hold. Then the uniform attractor $\mathscr{A}^{\varepsilon}$ for problem (1)-(4) converges to $\mathscr{A}^{0}$ of problem (5)-(8) in the limit $\varepsilon \rightarrow 0^{+}$in the following sense:

$$
\lim _{\varepsilon \rightarrow 0^{+}} \operatorname{dist}_{H}\left(\mathscr{A}^{\varepsilon}, \mathscr{A}^{0}\right)=0
$$

Proof. For $\varepsilon>0, u^{\varepsilon} \in \mathscr{A}^{\varepsilon}$, from (110)-(111), we obtain that there exists a complete bounded trajectory $\widehat{u}^{\varepsilon}(t)$ of (38), with some external force

$$
\widehat{f}^{\varepsilon}=\widehat{f}_{0}+\varepsilon^{-\rho} \widehat{f}_{1}\left(\frac{\cdot}{\varepsilon}\right) \in \mathscr{H}\left(f^{\varepsilon}\right),
$$

such that $\widehat{u}^{\varepsilon}(0)=u^{\varepsilon}$. 
We choose $L \geq 0$ such that

$$
\widehat{u}^{\varepsilon}(-L) \in \mathscr{A}^{\varepsilon} \subset B_{*} .
$$

From the equality

$$
u^{\varepsilon}=U_{\widehat{f}^{0}}(0,-L) \widehat{u}^{\varepsilon}(-L)
$$

and applying Lemma 12 with $t=0, \tau=-L$, we obtain

$$
\left\|u^{\varepsilon}-U_{\widehat{f}^{0}}(0,-L) \widehat{u}^{\varepsilon}(-L)\right\|_{H} \leq D \varepsilon^{1-\rho} e^{R L} .
$$

On the other hand, the set $\mathscr{A}^{0}$ attracts all sets $U_{\hat{f}^{0}}(t,-L) B_{*}$ uniformly when $\widehat{f}_{0} \in \mathscr{H}\left(f^{0}\right)$. Then, for all $\delta>0$, there exists some time $T=T(\delta) \geq 0$ which is independent on $L$, such that

$$
\operatorname{dist}_{H}\left(U_{\widehat{f}^{0}}(T-L,-L) \widehat{u}^{\varepsilon}(-L), \mathscr{A}^{0}\right) \leq \delta .
$$

Choosing $L=T$ and using (123)-(124), we readily get

$$
\begin{aligned}
\operatorname{dist}_{H}\left(u^{\varepsilon}, \mathscr{A}^{0}\right) \leq & \left\|u^{\varepsilon}-U_{\widehat{f}^{0}}(0,-T) \widehat{u}^{\varepsilon}(-T)\right\|_{H} \\
& +\operatorname{dist}_{H}\left(U_{\widehat{f}^{0}}(0,-T) \widehat{u}^{\varepsilon}(-T), \mathscr{A}^{0}\right) \\
\leq & D \varepsilon^{1-\rho} e^{R T}+\delta .
\end{aligned}
$$

Since $u^{\varepsilon} \in \mathscr{A}^{\varepsilon}$ and $\delta>0$ is arbitrary, taking the limit $\varepsilon \rightarrow 0^{+}$, we can prove the theorem.

\section{Acknowledgments}

Xin-Guang Yang was in part supported by the Young Teacher Research Fund of Henan Normal University (qd12104) and the Innovational Scientists and Technicians Troop Construction Projects of Henan Province (no. 114200510011). Jun-Tao Li was in part supported by the Natural Science Foundation of China (61203293), Foundation of Henan Educational Committee (2011B120005), Key Scientific and Technological Project of Henan Province (122102210131), and College Young Teachers Program of Henan Province (2012GGJS-063).

\section{References}

[1] J. Leray, "Essai sur les mouvements plans d'un liquide visqueux que limitentdes parois," Journal de Mathématiques Pures et Appliquées, vol. 13, pp. 331-418, 1934.

[2] J. Leray, "Sur le mouvement d'un liquide visqueux emplissant l'espace," Acta Mathematica, vol. 63, no. 1, pp. 193-248, 1934.

[3] E. Hopf, "Über die Anfangswertaufgabe für die hydrodynamischen Grundgleichungen," Mathematische Nachrichten, vol. 4, pp. 213-231, 1951.

[4] G. R. Sell, "Global attractors for the three-dimensional NavierStokes equations," Journal of Dynamics and Differential Equations, vol. 8, no. 1, pp. 1-33, 1996.

[5] A. Cheskidov and C. Foias, "On global attractors of the 3D Navier-Stokes equations," Journal of Differential Equations, vol. 231, no. 2, pp. 714-754, 2006.
[6] F. Flandoli and B. Schmalfuß, "Weak solutions and attractors for three-dimensional Navier-Stokes equations with nonregular force," Journal of Dynamics and Differential Equations, vol. 11, no. 2, pp. 355-398, 1999.

[7] P. E. Kloeden and J. Valero, "The weak connectedness of the attainability set of weak solutions of the three-dimensional Navier-Stokes equations," Proceedings of The Royal Society of London A, vol. 463, no. 2082, pp. 1491-1508, 2007.

[8] N. J. Cutland, "Global attractors for small samples and germs of 3D Navier-Stokes equations," Nonlinear Analysis: Theory, Methods \& Applications, vol. 62, no. 2, pp. 265-281, 2005.

[9] V. V. Chepyzhov and M. I. Vishik, "Evolution equations and their trajectory attractors," Journal de Mathématiques Pures et Appliquées, vol. 76, no. 10, pp. 913-964, 1997.

[10] V. V. Chepyzhov and M. I. Vishik, Attractors for Equations of Mathematical Physics, American Mathematical Society, Providence, RI, USA, 2002.

[11] M. I. Vishik and V. V. Chepyzhov, "Trajectory and global attractors of the three-dimensional Navier-Stokes system," Mathematical Notes, vol. 71, no. 2, pp. 177-193, 2002.

[12] A. V. Kapustyan and J. Valero, "Weak and strong attractors for the 3D Navier-Stokes system," Journal of Differential Equations, vol. 240, no. 2, pp. 249-278, 2007.

[13] O. V. Kapustyan, P. O. Kasyanov, and J. Valero, "Pullback attractors for a class of extremal solutions of the 3D Navier-Stokes system," Journal of Mathematical Analysis and Applications, vol. 373, no. 2, pp. 535-547, 2011.

[14] O. A. Ladyzhenskaya, The Mathematical Theory of Viscous Incompressible Flow, Gordon and Breach Science Publishers, New York, NY, USA, 1969.

[15] J. C. Robinson, Infinite-dimensional Dynamical Systems, Cambridge University Press, Cambridge, UK, 2001.

[16] G. R. Sell and Y. You, Dynamics of Evolutionary Equations, Springer, New York, NY, USA, 2002.

[17] R. Temam, Navier-Stokes Equations, Theory and Numerical Analysis, North-Holland, Amsterdam, The Netherlands, 1979.

[18] R. Temam, Infinite Dimensional Dynamical Systems in Mechanics and Physics, Springer, Berlin, Germany, 2nd edition, 1997.

[19] T. Caraballo and J. Real, "Attractors for 2D-Navier-Stokes models with delays," Journal of Differential Equations, vol. 205, no. 2, pp. 271-297, 2004.

[20] V. V. Chepyzhov, M. I. Vishik, and V. Pata, "Averaging of 2D Navier-Stokes equations with singularly oscillating forces," Nonlinearity, vol. 22, no. 2, pp. 351-370, 2009.

[21] M. I. Vishik and V. V. Chepyzhov, "The global attractor of a nonautonomous two-dimensional Navier-Stokes system with a singularly oscillating external force," Doklady Mathematics, vol. 413, no. 3, pp. 236-239, 2007.

[22] C. Foias and R. Temam, "The connection between the NavierStokes equations, dynamical systems, and turbulence theory," in Directions in Partial Differential Equations, pp. 55-73, Academic Press, New York, NY, USA, 1987.

[23] C. Foias and R. Temam, "Some analytic and geometric properties of the solutions of the evolution Navier-Stokes equations," Journal de Mathématiques Pures et Appliquées, vol. 58, no. 3, pp. 339-368, 1979.

[24] R. Rosa, "The global attractor for the D Navier-Stokes flow on some unbounded domains," Nonlinear Analysis: Theory, Methods \& Applications, vol. 32, no. 1, pp. 71-85, 1998.

[25] Y. Hou and K. Li, "The uniform attractor for the $2 D$ nonautonomous Navier-Stokes flow in some unbounded domain," 
Nonlinear Analysis: Theory, Methods \& Applications, vol. 58, no. 5-6, pp. 609-630, 2004.

[26] S. Lu, H. Wu, and C. Zhong, "Attractors for nonautonomous 2D Navier-Stokes equations with normal external forces," Discrete and Continuous Dynamical Systems A, vol. 13, no. 3, pp. 701-719, 2005.

[27] S. Lu, "Attractors for nonautonomous 2D Navier-Stokes equations with less regular normal forces," Journal of Differential Equations, vol. 230, no. 1, pp. 196-212, 2006.

[28] A. Miranville and X. Wang, "Attractors for nonautonomous nonhomogeneous Navier-Stokes equations," Nonlinearity, vol. 10, no. 5, pp. 1047-1061, 1997.

[29] X. Cai and Q. Jiu, "Weak and strong solutions for the incompressible Navier-Stokes equations with damping," Journal of Mathematical Analysis and Applications, vol. 343, no. 2, pp. 799809, 2008.

[30] X.-L. Song and Y.-R. Hou, "Attractors for the three-dimensional incompressible Navier-Stokes equations with damping," Discrete and Continuous Dynamical Systems A, vol. 31, no. 1, pp. 239-252, 2011.

[31] V. K. Kalantarov and E. S. Titi, "Global attractors and determining modes for the 3D Navier-Stokes-Voight equations," Chinese Annals of Mathematics B, vol. 30, no. 6, pp. 697-714, 2009.

[32] Y. Qin, X. Yang, and X. Liu, "Uniform attractors for a 3D nonautonomous Navier-Stokes-Voight Equations," In press.

[33] X. Yang, "Uniform attractors for the 2D non-autonomous Navier-Stokes Equations with damping," Acta Mathematica Scientia. In press.

[34] Y. Qin, X. Yang, and X. Liu, "Averaging of a 3D Navier-StokesVoight equation with singularly oscillating forces," Nonlinear Analysis: Real World Applications, vol. 13, no. 2, pp. 893-904, 2012. 


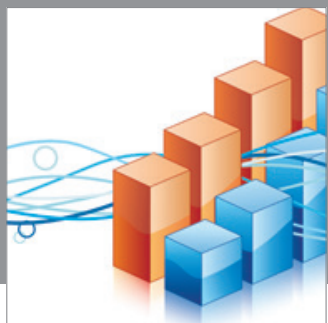

Advances in

Operations Research

mansans

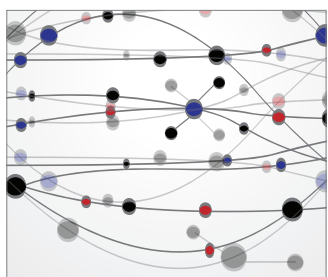

The Scientific World Journal
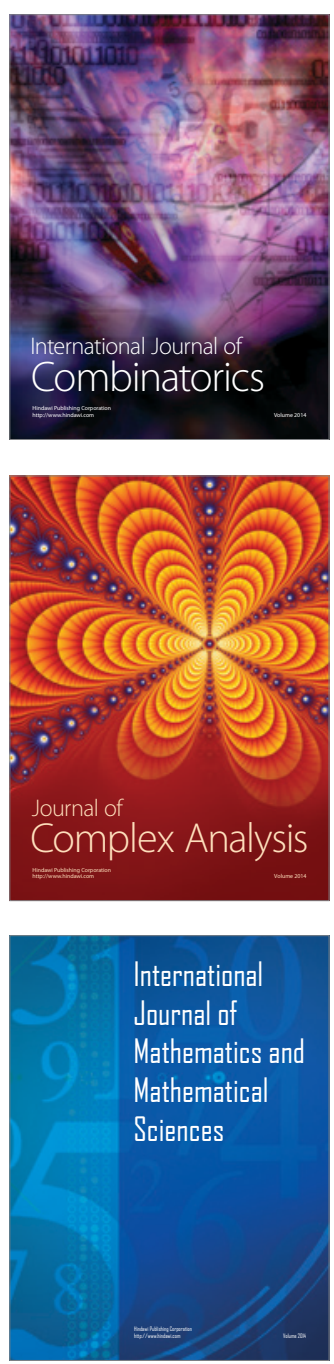
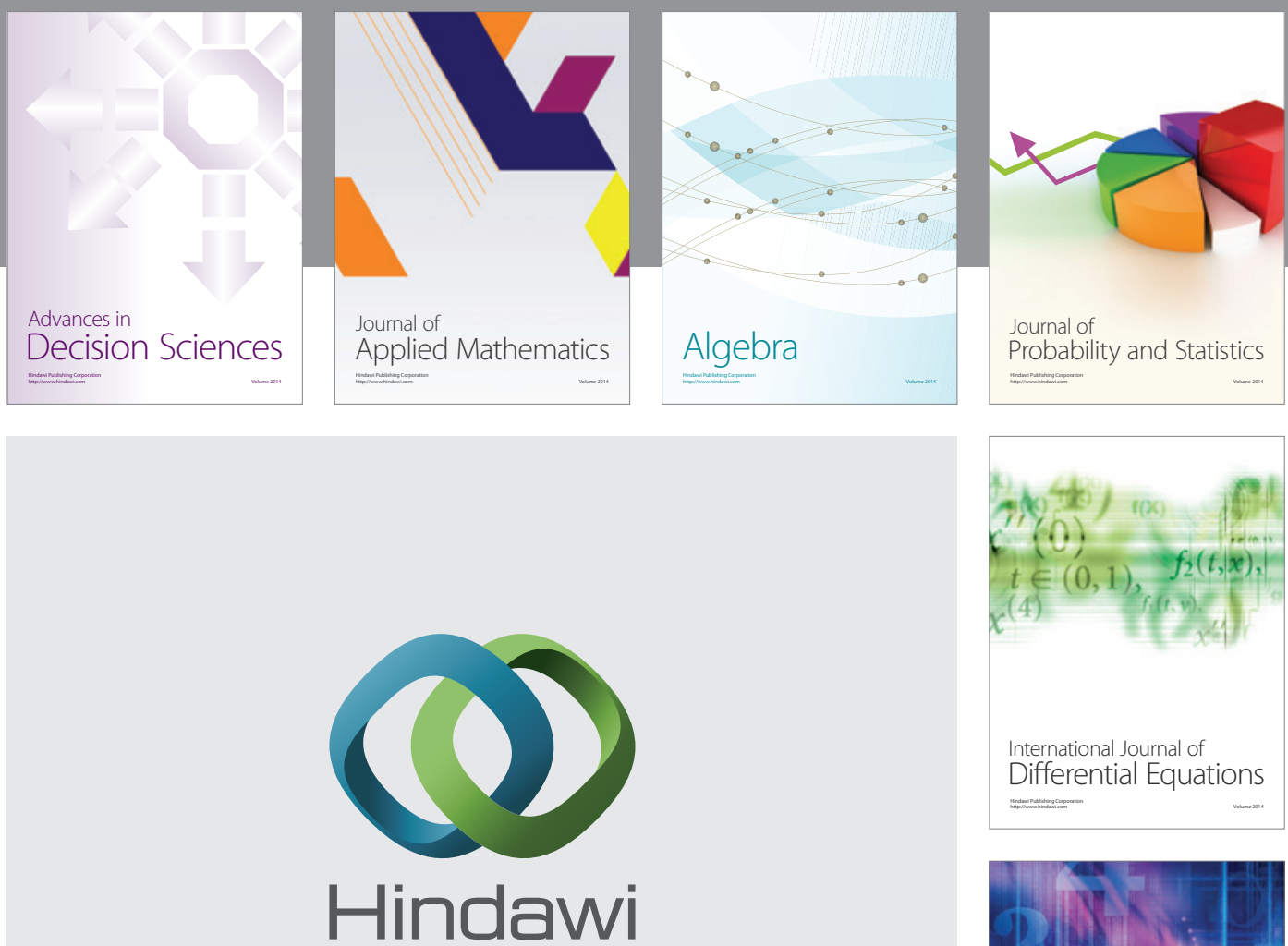

Submit your manuscripts at http://www.hindawi.com
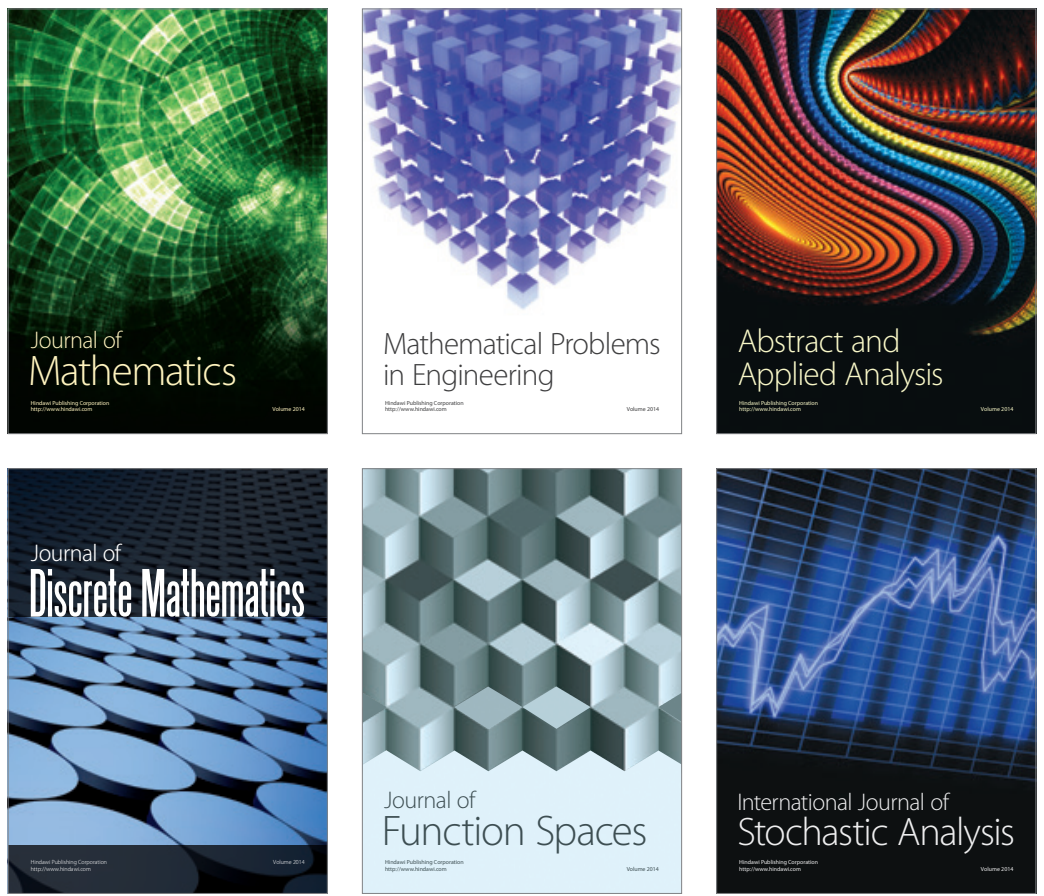

Journal of

Function Spaces

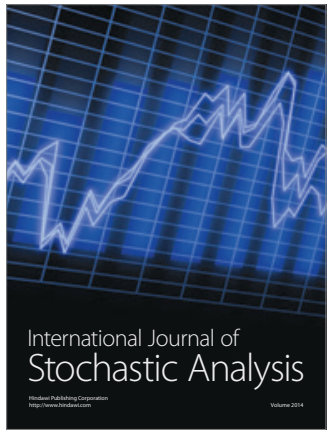

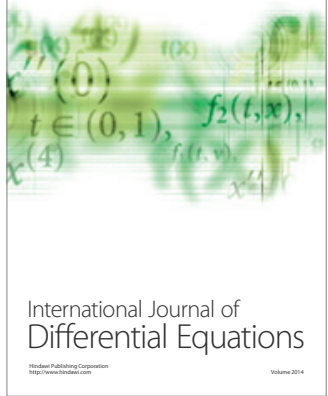
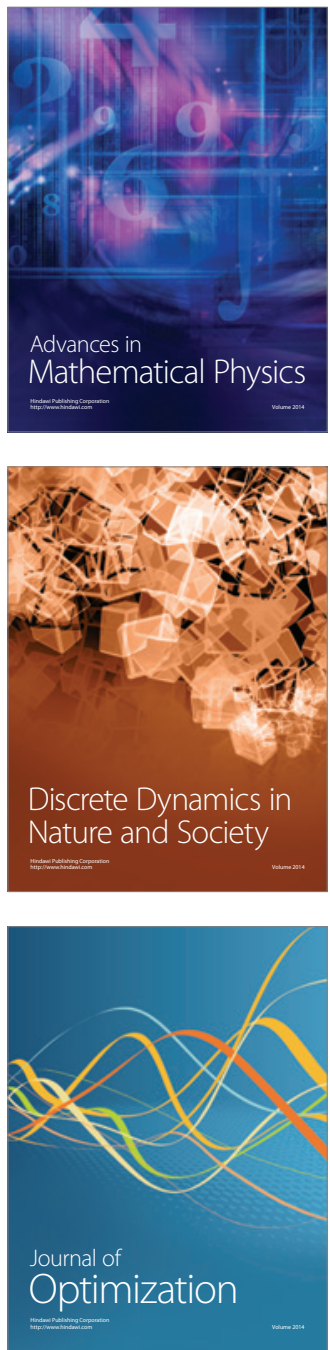